\title{
Synthesis of Germanium-Gallium-Tellurium (Ge-Ga-Te) ceramics by ball-milling and sintering
}

Mathieu Hubert, Elena Petracovschi, Xiang-Hua Zhang and Laurent Calvez*

Equipe Verres et Céramiques, UMR 6226 Institut des Sciences Chimiques de Rennes, Campus de Beaulieu, Avenue du Général Leclerc, 35042, Rennes, France

*laurent.calvez@univ-rennes1.fr

Tel: (33) 223236713

Fax: (33) 223235611

\begin{abstract}
In this paper, we present the preparation of a bulk material with a composition of $80 \mathrm{GeTe}_{2}-20 \mathrm{Ga}_{2} \mathrm{Te}_{3}$ by combining mechanosynthesis and sintering. This composition cannot be prepared by conventional melt/quenching technique. The progressive evolution of the powder during ball milling is followed by XRD and DSC analysis. The final powder obtained is highly crystalline but a glass transition temperature is observed, indicating the presence of some amorphous phase remaining, allowing for its efficient sintering. By hot pressing, a dense bulk material with a fine microstructure and a high electrical conductivity is obtained. The synthesis method described represents a simple and cost effective way to produce tellurium-based materials of desired dimension with potential applications for optical storage or thermoelectric devices.
\end{abstract}

\section{Introduction}

Due to their particular properties, tellurium-based materials are used within a large field of applications. For example, the semiconductor behavior of CdTe is exploited for the production of solar panels $[1,2]$, the rapid and reversible amorphous-to-crystalline phase change of some Ge-Sb-Te or TePd based films is used to produce memory chips or optical disks for data storage [3-8], and materials such as $\mathrm{Bi}_{2} \mathrm{Te}_{3}[9-11]$ or PbTe $[12,13]$ are reference materials for thermoelectric applications. Also, the high refractive index of some telluride glasses associated to their extended transparency in the far infrared up to 20-25 $\mu \mathrm{m}$ make them of paramount interest for the production of optical fibers for far infrared detection for medical or spatial purposes [14-17]. Yet, tellurium-based glasses are often unstable towards crystallization [18]. Therefore, their synthesis using the conventional melt/quenching technique is very demanding and represents a major limitation to their use. 
Amongst chalcogenide glasses, the $80 \mathrm{GeX}_{2}-20 \mathrm{Ga}_{2} \mathrm{X}_{3}\left(\right.$ or $\left.\mathrm{Ge}_{23.5} \mathrm{Ga}_{11.8} \mathrm{X}_{64.7}\right)$ composition from the GeGa-X systems, where $\mathrm{X}$ is a chalcogen elements (i.e. $\mathrm{X}=\mathrm{S}$, Se or Te), has revealed to be of particular interest. Indeed, the $80 \mathrm{GeS}_{2}-20 \mathrm{Ga}_{2} \mathrm{~S}_{3}[19,20]$ and $80 \mathrm{GeSe}_{2}-20 \mathrm{Ga}_{2} \mathrm{Se}_{3}[21,22]$ glasses show a controllable crystallization behavior, enabling the production of glass-ceramics with enhanced mechanical properties while keeping a good transparency in the infrared up to $11 \mu \mathrm{m}$ and $16 \mu \mathrm{m}$, respectively. Thus, the $80 \mathrm{GeTe}_{2}-20 \mathrm{Ga}_{2} \mathrm{Te}_{3}$ composition could logically appear as promising for the preparation of mechanically enhanced glass-ceramics with a transmission range extended further in the infrared, up to more than $20 \mu \mathrm{m}$. However, contrary to the Ge-Ga-S and Ge-Ga-Se systems, the $80 \mathrm{GeX}_{2}-20 \mathrm{Ga}_{2} \mathrm{X}_{3}$ composition in the Ge-Ga-Te system is located outside the glassy domain, as can be seen on Fig. 1. Thus, while amorphous $80 \mathrm{GeS}_{2}-20 \mathrm{Ga}_{2} \mathrm{~S}_{3}$ and $80 \mathrm{GeSe}_{2}-20 \mathrm{Ga}_{2} \mathrm{Se}_{3}$ glasses can be prepared by the conventional melt/quenching technique, amorphous $80 \mathrm{GeTe}_{2}-20 \mathrm{Ga}_{2} \mathrm{Te}_{3}$ cannot be obtained because the cooling rate achieved during the quenching step of the melt/quenching technique in sealed silica ampoule is insufficient to avoid the crystallization of the material, and the materials synthesized by this technique are fully crystallized and very brittle. This inherently prevents the study of $80 \mathrm{GeTe}_{2}-20 \mathrm{Ga}_{2} \mathrm{Te}_{3}$ materials for IR optical applications, despite the potential interest that could be expected from the trends observed by the studies of the $80 \mathrm{GeS}_{2}-20 \mathrm{Ga}_{2} \mathrm{~S}_{3}$ and $80 \mathrm{GeSe}_{2}-20 \mathrm{Ga}_{2} \mathrm{Se}_{3}$ compositions [19-22].

Recently, the possibility of preparing $80 \mathrm{GeSe}_{2}-20 \mathrm{Ga}_{2} \mathrm{Se}_{3}$ bulk glasses and glass-ceramics by combining mechanical alloying via ball milling (mechanosynthesis) and sintering has been proven [23]. This technique, which does not involve melting and quenching and thus avoids crystallization during quenching, allows the manufacture of bulk amorphous samples with higher dimensions as compared to those prepared by the melt/quenching technique. As the energy involved during mechanical milling is greater than that provided during melting, mechanosynthesis might also allow a broadening of the glassy domain and the synthesis of amorphous compositions that cannot be prepared by the conventional synthesis way [24]. Therefore, this synthesis way combining mechanical milling and sintering appears as a promising way to prepare amorphous $80 \mathrm{GeTe}_{2}-20 \mathrm{Ga}_{2} \mathrm{Te}_{3}$ which cannot be obtained by melt/quenching. The preparation of this material, its properties and potential applications, have been investigated in this paper. 


\section{Experimental Procedure}

\section{Powder synthesis}

Chalcogenide powders of composition $80 \mathrm{GeTe}_{2}-20 \mathrm{Ga}_{2} \mathrm{Te}_{3}$ were prepared by mechanical alloying by introducing stochiometric amounts of pure raw metallic germanium $(5 \mathrm{~N}$, Umicore), gallium $(6 \mathrm{~N}$, Cerac) and tellurium (5N, Umicore) in the form of solids chunks of about $2 \mathrm{~mm}$ of diameter in a tungsten carbide (WC) grinding jar containing $6 \mathrm{WC}$ milling balls. The amount of powder prepared was 20 grams, giving a ball-to-powder weight ratio of 19:1. The jar was introduced into a planetary ball mill (Retsch PM100) placed in a glove box under argon atmosphere to reduce potential contaminations by oxygen and water. Rotation cycles of $3 \mathrm{~min}$ at $300 \mathrm{rpm}$ were scheduled with direction reversal and a pause of 3 min between each cycle. Powder samples were regularly collected from the jar for analyses. After each sample collection at $20 \mathrm{~h}, 40 \mathrm{~h}, 60 \mathrm{~h}$ and $80 \mathrm{~h}$, the jar was resealed and the milling was pursued. The total duration of milling is $100 \mathrm{~h}$.

\section{Powder sintering}

The bulk sample was prepared by introducing 2 grams of the powder milled for $100 \mathrm{~h}$ in a stainless steel die of $20 \mathrm{~mm}$ inner diameter. The walls of the die were previously covered with a layer of boron nitride powder to avoid sticking of the chalcogenide powder. Boron Nitride powder was diluted in laboratory grade ethanol and the solution was applied with a paintbrush over the walls of the die. The die was then left to dry in air atmosphere, leaving a thin and uniform layer of BN powder on the surface. The die was introduced into a hot uniaxial press evacuated under primary vacuum. The temperature was raised up to $400{ }^{\circ} \mathrm{C}$ in $60 \mathrm{~min}$ (heating rate of $6^{\circ} \mathrm{C} / \mathrm{min}$ ) with a pressure applied of 2 tons. The temperature of $400^{\circ} \mathrm{C}$ corresponds to the maximum temperature that can be achieved on the hot press utilized. This temperature is higher than the glass transition temperature $\mathrm{Tg}$ of the powder sintered and the results presented hereafter show that it is enough for obtaining a good sintering. The temperature was kept at $400^{\circ} \mathrm{C}$ for $2 \mathrm{~h}$, and then slowly decreased to room temperature with a cooling rate of $4^{\circ} \mathrm{C} / \mathrm{min}$. The pressure was released after the $2 \mathrm{~h}$ sintering dwell, at the beginning of the cooling process.

\section{$\underline{\text { Materials characterization }}$}


The thermal properties of the powder taken out from the jar at different milling durations were analyzed by differential scanning calorimetry on $10 \mathrm{mg}$ of powder using a ramp of $10^{\circ} \mathrm{C} / \mathrm{min}$ (DSC Q20, TA Instruments). The glass transition temperature Tg and the crystallization temperature were defined as the intersect of the endothermic change in slope on the thermogram and the onset of the exothermic peak, respectively, with an accuracy of $\pm 1^{\circ} \mathrm{C}$.

The evolution of the powder with mechanosynthesis duration was analyzed by carrying out $\mathrm{X}$ Ray Diffraction (XRD) analysis using a PANalytical X'Pert Pro diffractometer (Cu Ka 1.5418 ̊́).

The microstructure of the bulk sample prepared was observed using SEM (FEG-SEM JEOL 7400F). The crystalline phases were analyzed by XRD analysis of a polished disc. The density of the sintered material was measured using Archimede's double weighing technique with an accuracy of \pm 0.03 $\mathrm{Kg} / \mathrm{m}^{3}$.

The measure of the resistivity $(\rho)$ was performed using the four-point probe technique with an applied current of $100 \mathrm{~mA}$. The accuracy of the measurement is $\pm 7 \%$. The electrical conductivity $\sigma$ was calculated from value of the resistivity $(\sigma=1 / \rho)$.

\section{Results}

\section{Powder characterization}

Fig. 2 shows the evolution of the XRD patterns measured on the powder as a function of the milling duration. After $20 \mathrm{~h}$ of milling, the XRD pattern shows sharp diffraction peaks, but a broad peak characteristic of the presence of some amorphous phase in the powder is also observed. It is important to emphasize that no amorphous phase can be obtained by the melt/quenching technique. The sharp diffraction peaks observed on the XRD spectrum correspond to the crystalline phases GeTe (ICDD card 00-047-1079), $\mathrm{Ga}_{2} \mathrm{Te}_{3}$ (ICDD card 00-035-1490) and $\mathrm{Ga}_{2} \mathrm{Te}_{5}$ (ICDD card 00-031-0549). Some peaks corresponding to a pure crystalline Ge phase (ICDD card 00-004-0545) are also observed, indicating that the germanium is not fully reacted after $20 \mathrm{~h}$ of milling. With increased milling duration, the peaks corresponding to the pure Ge progressively vanish, indicating a progressive microstructural evolution of the powder and the progressive incorporation of the germanium. Moreover, the patterns progressively flatten, indicating a gradual decrease in the amount of amorphous phase within 
the powder. After $100 \mathrm{~h}$ of milling, the Ge has fully reacted, and the XRD pattern of the powder only shows diffraction peaks corresponding to the GeTe, $\mathrm{Ga}_{2} \mathrm{Te}_{3}$ and $\mathrm{Ga}_{2} \mathrm{Te}_{5}$ phases.

As can be seen on the DSC measurements performed on the powder as a function of the milling time (Fig. 3), a glass transition temperature is observed at $180^{\circ} \mathrm{C}$ for the powder milled for $20 \mathrm{~h}$. This confirms the presence of an amorphous phase in the $80 \mathrm{GeTe}_{2}-20 \mathrm{Ga}_{2} \mathrm{Te}_{3}$ powder. For longer milling duration, the intensity of the crystallization peak located around $200^{\circ} \mathrm{C}$ progressively diminishes. The DSC measurements were carried out on the same amount of powder $(10 \mathrm{mg})$, for each sample collected after different milling duration. The thermodynamic phenomena observed can therefore be quantitatively compared to each other. The diminution in the intensity, i.e. the area under the crystallization peaks, is due to a diminution in the amount of the heat required to ensure the transition from amorphous to crystalline phases. The amounts of powder analyzed being the same, samples presenting lower values of crystallization peak intensities possessed lower initial amount of crystalline phase (thus less heat is necessary during the crystallization process). It can therefore be concluded that, with increased milling duration, the decreased intensities in the crystallization peaks observed in Fig 3. is a result of a lower amount of initial amorphous phase, meaning that progressive crystallization occurred as the milling duration increases. This observation is in agreement with the increase in crystallinity of the powder with increased milling time previously observed on the XRD analysis. However, it has to be emphasized that the observation of a crystallization peak even after $100 \mathrm{~h}$ of milling clearly indicates that a small amount of amorphous phase remains in the powder.

\section{$\underline{\text { Bulk sample characterization }}$}

The previous results showed the high crystallinity of the powder obtained after $100 \mathrm{~h}$ of milling. However, DSC measurements showed the presence of some residual amorphous phase. Heating the remaining vitreous phase above $\mathrm{Tg}$ allows reaching viscosities low enough to enable the sintering of the powder and ensure a good cohesion between the different grains by binding crystalline grains together. The powder obtained after $100 \mathrm{~h}$ of milling has thus been sintered by hot pressing, and the bulk material obtained is shown in Fig. 4-a. The sample, with a diameter of $20 \mathrm{~mm}$ and $0.75 \mathrm{~mm}$ thick has a density of $6.03 \mathrm{~g} . \mathrm{cm}^{-3}$. The sintered material presents good mechanical properties allowing its handling by operators without breakage, cracks or failure during the analyses. After polishing, the 
obtained material has a shiny metallic aspect, similar to glasses from the system Ge-Ga-Te obtained by melt/quenching [18]. However, the sintered sample does not present any optical transmission. Therefore, the $80 \mathrm{GeTe}_{2}-20 \mathrm{Ga}_{2} \mathrm{Te}_{3}$ composition shows no interest for optical applications in the infrared range. Nonetheless, numerous opaque Te-based materials are employed for other applications such as thermoelectric materials or rewritable discs, which are discussed in more details in the following section.

The SEM observation of the sintered material (Fig. 4-b and 4-c) confirms the important crystallinity of the sample. The images reveal the presence of crystals of about $1 \mu \mathrm{m}$. A very low residual porosity is observed, indicating a high densification rate during the sintering. It has to be noted that no contamination by the boron nitride powder employed during sintering was observed on the polished sample after visual and SEM observation, as well as XRD analysis.

Fig. 5 shows the XRD pattern measured on the powder before sintering and on the bulk $80 \mathrm{GeTe}_{2^{-}}$ $20 \mathrm{Ga}_{2} \mathrm{Te}_{3}$ ceramic. The sintered sample shows more intense and sharper diffraction peaks, indicating a crystal growth phenomenon during sintering. The crystalline phases $\mathrm{GeTe}, \mathrm{Ga}_{2} \mathrm{Te}_{3}$ and $\mathrm{Ga}_{2} \mathrm{Te}_{5}$ are present before and after sintering of the powder. The XRD pattern of the bulk ceramic also shows four additional crystalline peaks in comparison to the non-sintered powder. Therefore, an additional phase has precipitated during the sintering. This phase has been identified as pure metallic tellurium (ICDD card 01-085-0560). The precipitation of Te nanodots has been observed in other studies about sintering of tellurium-based powders [25].

The value of electrical resistivity measured on the bulk ceramic is $\rho=7.5 \times 10^{-6} \Omega . \mathrm{m}$, corresponding to a value of electrical conductivity of $\sigma=1.33 \times 10^{5} \mathrm{~S}^{-\mathrm{m}^{-1}}$.

\section{Discussion}

We demonstrated a simple and cost-effective way to produce bulk materials that cannot be produced by the melt/quenching technique. From the analyses performed and the characteristics of the sintered ceramic, it can be concluded that the powder produced by ball milling is highly crystalline, but a small amount of amorphous phase remains, as can be seen on the DSC curves in Fig 3. This phase accounts for the successful sintering of the powder at a temperature higher than its Tg. As no binder is added to the powder before its sintering, the production of a dense, bulk material after this step is due to the 
presence of this phase, which ensures the cohesion of the grains together within the ceramic. The initial aim of this study was the production of materials for optical infrared applications, telluriumbased chalcogenide glasses showing extended transparency up to $20 \mu \mathrm{m}$ in the infrared range $[14,18]$. Despite optical measurements performed on the sintered $80 \mathrm{GeTe}_{2}-20 \mathrm{Ga}_{2} \mathrm{Te}_{3}$ ceramic showed no optical transmission, the other properties measured on this material could make them interesting for different applications.

On the one hand, this ceramic contains microcrystals of GeTe and $\mathrm{Ga}_{2} \mathrm{Te}_{3}$. Bruns et al [26] for GeTe, and $\mathrm{Zhu}$ et al [27] for $\mathrm{Ga}_{2} \mathrm{Te}_{3}$, notably, have shown that these two phases have fast and reversible crystalline-amorphous-crystalline transformations and are consequently of great interest for phase change random access memory (PCRAM) devices. Furthermore, the synthesis way described in the present study allows for controlling the final geometry of the sintered sample, by adjusting the sintering parameters (amount of powder introduced into the die, geometry of the die), and devices could be sintered to the desired final dimensions. Therefore, the synthesis of crystals of GeTe and $\mathrm{Ga}_{2} \mathrm{Te}_{3}$ on the micron and submicron scale and their integration within bulk materials designed for PCRAM devices represents a potential application for germanium-gallium-tellurium ceramics prepared by ball milling and sintering. The ceramic prepared in this study contains a mix of different crystalline phases. It would be interesting to adjust the initial stoichiometry of the elements introduced in the milling jar in order to precipitate one specific phase. This work will be part of a subsequent study.

On the other hand, the electrical conductivity of the sintered ceramic could represent a major advantage for thermoelectric applications. In fact, the efficiency of thermoelectric devices is defined by its zT value, calculated from the equation $z T=\sigma S^{2} \mathrm{~T} / \kappa$ where $\sigma$ is the electrical conductivity, $\mathrm{S}$ is the Seebeck coefficient, $\mathrm{T}$ is the temperature, and $\kappa$ is the thermal conductivity [28]. It appears from this equation that high values of electrical conductivity and low values of thermal conductivity are sought for high zT values. One of the reference materials for thermoelectric applications near room temperature is $\mathrm{Bi}_{2} \mathrm{Te}_{3}$. Recent studies, notably by Zhao et al. [29, 30], have demonstrated the possibility to produce $\mathrm{Bi}_{2} \mathrm{Te}_{3}$ bulk materials by sintering with SPS a powder prepared by mechanical alloying (ball milling). They showed that the final properties of the material are dependent on the 
sintering parameters (temperature and pressure [29], annealing [30]). The electrical conductivities measured on their materials, depending on the sintering parameters, range from 0.7 to $1.4 \times 10^{5} \mathrm{~S} . \mathrm{m}^{-1}$ at room temperature. The electrical conductivity of the sintered $80 \mathrm{GeTe}_{2}-20 \mathrm{Ga}_{2} \mathrm{Te}_{3}$ ceramic $(\sigma=1.33$ $\mathrm{x} 10^{5} \mathrm{~S} . \mathrm{m}^{-1}$ at room temperature is therefore comparable to the electrical conductivity of the reference $\mathrm{Bi}_{2} \mathrm{Te}_{3}$ when prepared by ball milling and sintering.

The thermal conductivity $\kappa$ can be divided into two parts: the electronic and the lattice part. In the case of our study, it was not possible to measure the thermal conductivity of the sintered material. However, studies have shown that the lattice part of the thermal conductivity is controlled by diffuse and inelastic scattering processes at interfaces and can be reduced by introduction interfaces within the material $[31,32]$. The importance of an effective interface electron-phonon carrier charge transfer in the observed thermoelectric properties for $\mathrm{Bi}_{2} \mathrm{Te}_{3}$ materials was confirmed in a study by Kaddouri et al [33]. The ceramic prepared in this study shows a fine microstructure with a grain size of about $1 \mu \mathrm{m}$ (Fig 4), which implies a large number of interfaces, favoring low lattice contribution of the thermal conductivity. This would therefore tend to decreased thermal conductivities, thus increased zT and better thermoelectric properties.

It is worth noting that a reduction of the grain size to some tens of nanometers within the sintered material would be beneficial for thermoelectric applications. As mentioned previously, the efficiency of thermoelectric materials is dependent on the thermal conductivity of the material and consequently to the phenomena at the interfaces within the material. Increasing the interface area would lead to reduced thermal conductivity and more efficient thermoelectric properties [31,32]. The amount of interface is related to the microstructure of material, and greater interface can be achieved by a reduction of the grain size within the sintered sample. Such a reduction of the grain size can be obtained by using an initial powder with a finer grain size distribution, by varying the ball milling parameters and/or by sieving. One key factor in the grain size of the final bulk microstructure is grain growth during sintering. Grain growth is favored with prolonged sintering duration at higher temperatures [34]. To have finest possible grain size, it thus appears necessary to reduce sintering time and temperature. In that respect, the Spark Plasma Sintering (SPS) technique could be of great interest, as it allows for very short sintering times and reduced temperatures, and can suppress grain growth 
phenomena to some extent [35]. Moreover, this technique has already proven being efficient for the preparation of chalcogenide glasses and glass-ceramics [23], as well as for thermoelectric materials $[25,36,37]$. In order to obtain materials with finer grain microstructure and increase their interest for thermoelectric applications, Spark Plasma Sintering therefore appears as a technique of choice to sinter the germanium-gallium-tellurium powders prepared by ball milling.

\section{Conclusion}

The simple and cost effective technique presented in this study, combining mechanosynthesis and hot pressing, opens the way towards the production of germanium-gallium-tellurium ceramics that cannot be prepared by the conventional melt/quenching technique. By using ball milling and hot pressing, we demonstrated the possibility of producing a chalcogenide polycrystalline material with a starting composition of $80 \mathrm{GeTe}_{2}-20 \mathrm{Ga}_{2} \mathrm{Te}_{3}$. Ball milling of the raw starting elements yield a highly crystalline powder with a small amount of amorphous phase, while no amorphous phase is obtained when using the conventional melt/quenching technique. This remaining vitreous phase allowed for the good sintering of the powder. The bulk material obtained, while being optically opaque, possesses properties that make it interesting for other applications. On the one hand, the high electrical conductivity and the fine microstructure of the sintered ceramic are major advantages for thermoelectric applications. On the other hand, the $\mathrm{GeTe}$ and $\mathrm{Ga}_{2} \mathrm{Te}_{3}$ crystalline phases identified within the material can find applications in PCRAM applications due to their rapid crystallineamorphous-crystalline transitions. The microstructure of the material is a key parameter for these applications and it has been demonstrated that reducing grain size is essential. In that respect, using Spark Plasma Sintering appears as a promising way to produce germanium-gallium-tellurium ceramics with enhanced thermoelectric properties.

\section{Acknowledgements}

This work has been supported by the French DGA (Délégation Générale pour l'Armement), contract number REI $\mathrm{N}^{\circ} 08 \mathrm{c} 0055$.

\section{References}


1. A. Bosio, R. Nicola, S. Mazzamuto and V. Canevari, 'Polycrystalline CdTe thin films for photovoltaic applications', Prog. Cryst. Growth Ch. 52 [4] 247-279 (2006)

2. X. Mathew, G.W. Thompson, V.P. Singh, J.C. McClure, S. Velumani, N.R. Mathews and P.J. Sebastian, 'Development of CdTe thin films on flexible substrates-a review', Sol. Energ. Mat. Sol. C., 76 [3] 293-303 (2003)

3. M. Boniardi, D. Ielmini, I. Tortorelli, A. Redaelli, A. Pirovano, M. Allegra, M. Magistretti, C. Bresolin, D. Erbetta, A. Modelli, E. Varesi, F. Pellizzer, A.L. Lacaita and R. Bez, 'Impact of $\mathrm{Ge}-\mathrm{Sb}-\mathrm{Te}$ compound engineering on the set operation performance in phase-change memories', Solid State Electron. 58 [1] 11-16 (2011)

4. H.Y. Cheng, K.F. Kao, C.M. Lee and T.S. Chin, 'Crystallization kinetics of Ga-Sb-Te films for phase change memory', Thin Solid Films, 516 [16] 5513-5517 (2008)

5. S.S. Lin, 'Doped Ge-Sb-Te phase-change materials for reversible phase-change optical recording', Ceram. Int., 33 [7] 1161-1164 (2007)

6. R. Shayduk, R. and W. Braun, 'Epitaxial films for Ge-Sb-Te phase change memory', J. Cryst. Growth, 311 [7] 2215-2219 (2009)

7. G.F. Zhou, 'Materials aspects in phase change optical recording', Mat. Sci. Eng. A-Struct. 304$306[0] 73-80(2001)$

8. G.F. Zhou, B.A.J. Jacobs and W. van Es-Spiekman, 'Laser-induced crystallization in Ge-SbTe optical recording materials', Mat. Sci. Eng. A-Struct. 226-228 [0] 1069-1073 (1997)

9. E. Koukharenko, N. Fréty, V.G. Shepelevic and J.C. Tedenac, 'Thermoelectric properties of Bi2Te3 material obtained by the ultrarapid quenching process route', J. Alloy. Compd., 299 [1-2] 254-257 (2000)

10. S. Miura, Y. Sato, K. Fukuda, K. Nishimura and K. Ikeda, 'Texture and thermoelectric properties of hot-extruded Bi2Te3 compound', Mat. Sci. Eng. A-Struct. 277 [1-2] 244-249 (2000)

11. S.K. Mishra, S. Satpathy and O. Jepsen, 'Electronic structure and thermoelectric properties of bismuth telluride and bismuth selenide', J. Phys.-Condens. Mat. 9 [2] 461 (1997) 
12. H. Beyer, J. Nurnus, H. Bottner, A. Lambrecht, E. Wagner and G. Bauer, 'High thermoelectric figure of merit $\mathrm{ZT}$ in $\mathrm{PbTe}$ and $\mathrm{Bi} 2 \mathrm{Te} 3$-based superlattices by a reduction of the thermal conductivity', Physica E. 13 [2-4] 965-968 (2002)

13. Y. Gelbstein, Z. Dashevsky and M.P. Dariel, 'High performance n-type PbTe-based materials for thermoelectric applications', Physica B 363 [1-4] 196-205 (2005)

14. S. Danto, P. Houizot, C. Boussard-Plédel, X.H. Zhang, F. Smektala and J. Lucas, 'A Family of Far-Infrared-Transmitting Glasses in the Ga-Ge-Te System for Space Applications', Adv. Funct. Mat. 16 [14] 1847-1852 (2006)

15. V.S. Shiryaev, C. Boussard-Plédel, P. Houzot, T. Jouan, J.L. Adam and J. Lucas, 'Single-mode infrared fibers based on TeAsSe glass system', Mat. Sci. Eng. B-Solid, 127 [2-3] 138-143 (2006)

16. P. Lucas, D. Lecoq, C. Juncker, J. Collier, D.E. Boesewetter, C. Boussard-Plédel, B. Bureau and M. Riley, 'Evaluation of Toxic Agent Effects on Lung Cells by Fiber Evanescent Wave Spectroscopy', Appl. Spectrosc. 59 [1] 1-9 (2005)

17. J.S. Sanghera, L.B. Shaw and I.D. Aggarwal, 'Applications of chalcogenide glass optical fibers', CR Acad. Sci. II. C. 5 [12] 873-883 (2002)

18. B. Bureau, S. Danto, H.L. Ma, C. Boussard-Plédel, X.H. Zhang and J. Lucas, 'Tellurium based glasses: A ruthless glass to crystal competition', Solid State Sci. 10 [4] 427-433 (2008)

19. C. Lin, L. Calvez, M. Rozé, H. Tao, X.H. Zhang and X. Zhao, 'Crystallization behavior of 80GeS2 · 20Ga2S3 chalcogenide glass', Appl. Phys. A-Matter. 97 [3] 713-720 (2009)

20. C. Lin, S. Dai, C. Liu, B. Song, Y. Xu, F. Chen and J. Heo, 'Mechanism of the enhancement of mid-infrared emission from GeS2-Ga2S3 chalcogenide glass-ceramics doped with Tm3+', Appl. Phys. Lett. 100 [23] 231910 (2012)

21. M. Rozé, L. Calvez, M. Hubert, P. Toupin, B. Bureau, C. Boussard-Plédel and X.H. Zhang, 'Molded Glass-Ceramics for Infrared Applications', Int. J. Appl. Glass Sci. 2 [2] 129-136 (2011) 
22. M. Rozé, L. Calvez, Y. Ledemi, M. Allix, G. Matzen and X.H. Zhang, 'Optical and Mechanical Properties of Glasses and Glass-Ceramics Based on the Ge-Ga-Se System', $J$. Am. Ceram. Soc. 91 [11] 3566-3570 (2008)

23. M. Hubert, G. Delaizir, J. Monnier, C. Godart, H.L. Ma, X.H. Zhang and L. Calvez, 'An innovative approach to develop highly performant chalcogenide glasses and glass-ceramics transparent in the infrared range', Opt. Express 19 [23] 23513-23522 (2011)

24. C. Suryanarayana, 'Mechanical alloying and milling', Prog. Mat. Sci. 46 [1] 1-184 (2001)

25. B. Poudel, Q. Hao, Y. Ma, Y. Lan, A. Minnich, B. Yu, X. Yan, D. Wang, A. Muto, D. Vashaee, X. Chen, J. Liu, M.D. Dresselhaus, G. Chen and Z. Ren, 'High-Thermoelectric Performance of Nanostructured Bismuth Antimony Telluride Bulk Alloys', Science 320 [5876] 634-638 (2008)

26. G. Bruns, P. Merkelbach, C. Schlockermann, M. Salinga, M. Wuttig, T. D. Happ, J. B. Philipp and M. Kund, 'Nanosecond switching in GeTe phase change memory cells', Appl. Phys. Lett. 95 [4] 043108 (2009)

27. H. Zhu, Y. Jiang, X. Yidong and L. Zhiguo, 'Ga2Te3 phase change material for low-power phase change memory application', Appl. Phys. Lett. 97 [8] 083504 (2010)

28. G.J. Snyder and E.S. Toberer, 'Complex thermoelectric materials' Nature Mat. 7 [2] 105-114 (2008)

29. L.D Zhao, B.P. Zhang, J.F. Li, M. Zhou and W.S. Liu, 'Effects of process parameters on electrical properties of n-type Bi2Te3 prepared by mechanical alloying and spark plasma sintering' Physica B 400 [1-2] 11-15 (2007)

30. L.D Zhao, B.P. Zhang, W.S. Liu, H.L. Zhang and J.F. Li 'Effects of annealing on electrical properties of n-type Bi2Te3 fabricated by mechanical alloying and spark plasma sintering' $J$. Alloy. Compd 467 [1-2] 91-97 (2009)

31. G. Chen, 'Thermal conductivity and ballistic-phonon transport in the cross-plane direction of superlattices', Phys. Rev. B. 57 [23] 14958-14973 (1998) 
32. M.S. Dresselhaus, G. Chen, M.Y. Tang, R.G. Yang, H. Lee, D.Z. Wang, Z.F. Ren, J.P. Fleurial and P. Gogna, 'New Directions for Low-Dimensional Thermoelectric Materials', $A d v$. Mater. 19 [8] 1043-1053 (2007)

33. H. Kaddouri, S. Bénet, S. Charar, M. Makowaska-Janusik, J.C. Tedenac and I.V. Kityk, 'Simulation of thermoelectric properties of bismuth telluride single crystalline films grown on Si and SiO2 surfaces' Phys. Rev. B 62 [24] 17108-17114 (2000)

34. F.X. Gil, D. Rodriguez and J.A. Planell, 'Grain growth kinetics of pure titanium', Scripta Metall. Mater. 33 [8] 1361-1366 (1995)

35. G. Zhang, Q. Zhang, S. Chen, W. Liu, K. Lukas, X. Yan, H. Wang, D. Wang, C. Opeil and G. Chen, 'Suppression of grain growth by additive in nanostructured p-type bismuth antimony tellurides' Nano Energy 1 [1] 183-189 (2012)

36. M. Scheele, N. Oeshler, K. Meier, A. Kornowski, C. Klinke and H. Weller, 'Synthesis and Thermoelectric Characterization of Bi2Te3 Nanoparticles', Adv. Funct. Mater. 19 [21] 3476$3483(2009)$

37. X. Yan, G. Joshi, W. Liu, Y. Lan, H. Wang, S. Lee, J.W. Simonson, S.J. Poon, T.M. Tritt, G. Chen and Z.F. Ren, 'Enhanced Thermoelectric Figure of Merit of p-Type Half-Heuslers', Nano Letters 11 [2] 556-560 (2011) 


\section{Captions}

Figure 1: Glassy domain for the Ge-Ga-X systems with $X=S, S e, T e ~[18,22,25]$

Figure 2: XRD patterns measured on the powder as a function of the milling duration

Figure 3: DSC curves measured on the powder as a function of the milling duration

Figure 4: a) Bulk material obtained by sintering of the powder milled for $100 \mathrm{~h}, \mathrm{~b}$ ) and c) SEM observation of the microstructure

Figure 5: XRD pattern of the powder before sintering and of the sintered material 